ongoing quality assurance of syphilis testing; (4) procurement mechanisms supporting high-quality and affordable syphilis test kits and supplies; and (5) enhanced national reporting of syphilis cases.

Conclusion This first-ever Regional Guidance on syphilis testing will set standards on which future country assessments will be evaluated.

\section{P09.37 SYPHILIS RAPID TEST VALIDATION FOR TREPONEMAL DIAGNOSIS IN GUATEMALA, 2013-2014}

${ }^{1} \mathrm{E}$ Arana Flora, ${ }^{2} \mathrm{~L}$ Castillo-Signor, ${ }^{2} \mathrm{P}$ Marchorro, ${ }^{3} \mathrm{~V}$ Girón, ${ }^{2} \mathrm{~A}$ Lopez, ${ }^{2} \mathrm{C}$ Escobar, ${ }^{1} \mathrm{C}$ Vargas, ${ }^{1} \mathrm{R}$ Mendizabal-Burastero, 'S Morales-Miranda*. ${ }^{1} H I V$ Unit, Centers for Health Studies, Universidad Del Valle de Guatemala; ${ }^{2}$ National Health Laboratory, Ministry of Health, Guatemala; ${ }^{3}$ HIV National Program, Ministry of Health, Guatemala

\subsection{6/sextrans-2015-052270.421}

Background International guidelines for syphilis testing recommend non-treponemal tests, and confirmation by more complex treponemal test such as Treponema pallidum haemagglutination (TPHA), enzyme-linked immunosorbent assay (ELISA) or others. Similar to HIV rapid tests, rapid treponemal tests can improve point-of-care testing in laboratories with limited capacities. We present main results of a validation for treponemal diagnosis performed in Guatemala.

Methods A syphilis rapid test laboratory validation was performed in two phases during 2013-2014. First stage was performed using frozen serums from National Health Laboratory and second stage was performed using whole blood from pregnant women from peripheral health services from Guatemala City. Four treponemal rapid tests were evaluated in the first stage: Determine TP (DetTP), SD Syphilis (SDSyp), Omega Visitect (OVis), and Rapid Syphilis (RSyp). Only two tests were evaluated in the second stage: DetTP and SDSyp. For confirmation, TPHA was used for diagnosis of treponemal antibodies in serum and treponemal ELISA for plasma.

Results A total of 460 serums were evaluated in first stage and 432 whole blood samples in second stage. Compared to TPHA, sensitivity ranged from $87.8 \%$ for RSyp to $99.2 \%$ in DetTP in serum and $100 \%$ for SDSyp and DetTP in whole blood. Specificity was lower, ranging from $74.8 \%$ for DetTP to $87.7 \%$ for SDSyp in serum and $100 \%$ for SDSyp and DetTP in whole blood.

Conclusion Rapid treponemal tests are a useful diagnostic tool in syphilis. Sensibility was high enough to be used as a screening test, with good results in whole blood samples. Low prevalence of syphilis in the validation contributed to poor positive predictive values. The use of a reverse algorithm might be useful for implementation of this test in Guatemala.

Disclosure of interest statement We declare that we have no conflicts of interest.

\section{P09.38 SYPHILIS PREVALENCE AND RISK BEHAVIOUR AMONG PEOPLE LIVING WITH HIV IN MANAGUA, NICARAGUA: BSS-2009, BSS-2014 AND STI SENTINEL SURVEILLANCE (VICITS) 2014}

L Romero, I Loya-Montiel, A Solorzano, S Morales-Miranda. HIV Unit, Center for Health Studies, Universidad Del Valle de Guatemala

10.1136/sextrans-2015-052270.422
Background In Nicaragua, 9,739 HIV cases have been reported during 1987-2014. Two Central American Behavioural Surveillance Survey (BSS) among people living with HIV (PLHIV) to estimate prevalence of STI and risk behaviour have been conducted. Since 2012, the STI Sentinel Surveillance Strategy (VICITS) has offered STI diagnosis/treatment and behavioural intervention for PLHIV in public health sites. We present sociodemographic/behavioural and STI prevalence among PLHIV from BSS (2009 and 2014) and VICITS databases.

Methods For the BSS, we used a convenience sample of PLHIV seeking HIV services at Roberto Calderon Hospital (RCH) in Managua, from September-December 2009 and January-March 2014. For VICITS, PLHIV seeking services at RCH during 2014 were included. BSS information was obtained through Audio Computer-Assisted Self-Interview. VICITS data were abstracted from VICITS information system. Blood samples were collected for Syphilis testing according to national guidelines. Data were analysed using STATA v13.0.

Results Each BSS survey recruited 200 PLHIV (male 53.5\% BSS2009; 74.0\% BSS-2014). VICITS recruited 146 PLHIV $(85.7 \%$ male). Median age was 34 years old for all three data sources. One-third reported being married/living together in BSS-2009 and VICITS (44\%, BSS-2014). Alcohol use in the last month was reported by $33.2 \%$ in BSS-2009 and 50\% in BSS-2014 and VICITS. Condom use in the last sex with any partner was $60.0 \%$ in BSS-2009 and $75.5 \%$ in BSS-2014 and VICITS. Prevalence of syphilis was $11.6 \%$ (95\% CI: 7.5-16.8) in BSS-2009, $10.0 \%$ (95\% CI: $6.2-15.0$ ) in BSS-2014 and 16.7\% (95\% CI: 8.1-19.8) in VICITS.

Conclusion Although socio-demographic/behavioural data of PLHIV in BSS and VICITS were similar, syphilis prevalence was higher among PLHIV enrolled in VICITS. Our results indicate that VICITS strategy can provide valuable information about sexual behaviour of PLHIV. Our findings suggest the use of programmatic data in lieu of complex and expensive surveys to monitor trends in STI prevalence and sexual behaviour.

Disclosure of interest We declare that we have no conflicts of interest.

\section{P09.39 HIV/SYPHILIS PREVALENCE AND RISK BEHAVIOURS AMONG TRANSGENDER WOMEN AND MEN WHO HAVE SEX WITH MEN IN NICARAGUA: SENTINEL SURVEILLANCE AND STI CONTROL (VICITS), 2014}

R Gutiérrez, L Romero, I Loya-Montiel, A Solorzano, P Rosales, S Morales-Miranda*. HIV Unit of Center for Health Studies, Universidad Valle de Guatemala

\subsection{6/sextrans-2015-052270.423}

Background Nicaragua has a HIV concentrated epidemic, with 9.7\% among men who have sex with men and transgender women (MSM/TGW). Since 2011, Sentinel Surveillance and STI Control (VICITS) strategy has been implemented in public health clinics for key populations to provide STI diagnosis/treatment and behavioural change interventions. We describe risk sexual behaviours and HIV/syphilis prevalence among MSM/ TGW who attended at least one of two VICITS clinics.

Methods We included in the analysis: Men, $\geq 18$ years old (yo), who reported having sex with men in the last year, who attended one of the two selected VICITS clinics in Managua (Pedro Altamirano Hospital) or Granada (Sinforoso Bravo Hospital), from 1 January 2014 through 31 December 2014. Blood samples were collected for HIV and syphilis testing according to 